\title{
MSINR based Precoding Design for Non-Regenerative MIMO Relay System
}

\author{
Tao Zeng ${ }^{\dagger}$, Qingchun Chen ${ }^{\dagger}$, Pei Xiao ${ }^{\ddagger}$, Jinsong $\mathrm{Wu}^{*}$ \\ ${ }^{\dagger}$ Southwest Jiaotong University, Chengdu, 610031, China \\ $\ddagger$ University of Surrey, Guildford, Surrey, GU2 7XH, United Kingdom \\ ${ }^{*}$ Bell Laboratories, Shanghai 201206, China \\ lilac.15@163.com,qcchen@home.swjtu.edu.cn,p.xiao@surrey.ac.uk,wujs@ieee.org.
}

\begin{abstract}
The maximum signal-to-interference plus noise ratio (MSINR) design criterion is proposed in this paper to maximize the geometric product of signal-to-interference plus noise ratio (GEOM-SINR) in non-regenerative MIMO relay system. It is shown that the optimal MSINR based precoding at relay will diagonalize the equivalent source-relay-destination channel into parallel sub-channels, and the MSINR based MIMO relay precoding design will be transformed into the MSINR criterion based power allocation among multiple sub-channels. Simulation results are presented to corroborate the MSINR-based MIMO relay precoding design. It is unveiled that, compared with the existing maximal mutual information (MMI) based MIMO relay precoding design and the minimal mean square error (MMSE) based MIMO relay precoding design, MSINR-based MIMO relay precoding design is able to achieve a better tradeoff between the communication reliability and the realized ergodic capacity.
\end{abstract}

Index Terms-Non-regenerative relay, MIMO relay precoding, MSINR.

\section{INTRODUCTION}

It is known that MIMO technique could be utilized to enhance the spectral efficiency of wireless communication [1]. Meanwhile, relaying technique provides an effective means to circumvent the detrimental fading effects and path loss degradation for wider area wireless communication coverage and better communication quality. Relaying technique also finds applications in different wireless communication systems. By combining the advantages of both MIMO and relaying techniques, MIMO relaying technique offers an effective means to facilitate wideband wireless communication [2].

MIMO relay could be categorized into the non-regenerative relay and the regenerative relay. In the non-regenerative case, the MIMO relay will amplify and forward the received signal from the source to the destination. Since the non-regenerative MIMO relay is able to exploit more diversity gain than its regenerative counterpart given the same multiplexing gain [3], the non-regenerative MIMO relay precoding design has attracted many research interests in recent years. Previous research results unveil that reasonably devised precoding at the non-regenerative relay will benefit from significant performance improvement [3]- [6]. So far, different MIMO relay

This work was supported by the National Basic Research Program of China (973 Program No. 2012CB316100), the NSFC under grant No. $61032002 / 60872013$ and the Young Innovative Research Team of Sichuan Province under grant No.2011JTD0007. precoding design criteria have been proposed in the literature. For example, the maximum mutual information (MMI) based MIMO relay precoding design was addressed in [3]- [4], and the minimum mean square error (MMSE) based MIMO relay precoding design was proposed in [5]- [6]. Between these two MIMO relay precoding schemes, the MMSE-based MIMO relay precoding has higher reliability, while the MMI-based MIMO relay precoding may achieve higher ergodic capacity. How to realize a reasonable tradeoff between the reliability and the ergodic capacity via MIMO relay precoding design is the main concern of this paper.

Recently, a unified framework for optimizing nonregenerative MIMO relay communication systems is addressed in [9]. It is shown that, via joint source-relay precoding design, the source-relay-destination channel can be diagonalized into parallel single SISO channels, and the optimal source-relay precoding design problem will be transformed into the issues of power loading among multiple SISO channels. Motivated by the work in [9], a maximal signal to interference plus noise ratio (MSINR) based MIMO relay precoding design is investigated in this paper, wherein only the relay needs to participate in the precoding process. In fact, such a setup can simplify the system requirement, since only the relay needs to collect the necessary channel state information to realize the non-regenerative MIMO relay precoding. In this context, the geometric product of the SINR (GEOM-SINR) is used as the optimization metric. According to the majorization theory [7], it is proved that the optimal MSINR based MIMO precoding at relay will diagonalize the equivalent source-relay-destination channel into parallel sub-channels as well, and the MSINR based MIMO relay precoding design can be transformed into the MSINR criterion based power allocation among multiple sub-channels. By using the negative GEOM-SINR as the optimization objective and considering the transmit power constraint at relay, the optimal power allocation becomes a convex optimization problem, which could be readily solved by using the Lagrangian multiplier method. Simulation results are presented to corroborate the MSINR-based MIMO relay precoding design. Our analysis unveils that, compared with the MMI based MIMO relay precoding design and the MMSE based MIMO relay precoding design, MSINR-based MIMO relay precoding design is able to achieve a better tradeoff between the communication reliability and the realized er- 
godic capacity. The remainder of the paper is organized as follows. The non-regenerative MIMO relay communication model with relay precoding only is presented in Section II. The MSINR based MIMO relay precoding design is described in Section III. Numerical results are presented to validate the MSINR based MIMO relay precoding design in Section IV. Preliminary discussions on the reason why the proposed MSINR based MIMO relay precoding design offers a better tradeoff between the reliability and the ergodic capacity are heuristically explicated by the power allocation characteristics in Section IV as well. Finally some discussion are presented to conclude this paper in Section V.

The following notations are used throughout the paper: Matrices and vectors are written in bold uppercase and lowercase letters, respectively. $\operatorname{tr}(\mathbf{X})$ represents the trace of matrix $\mathbf{X}$ ; $\mathbf{X}^{H}$ denotes the conjugate transpose of $\mathbf{X} ;[\mathbf{X}]_{i, j}$ denotes the $i$-th row and the $j$-th column entry; $E[\cdot]$ represents the statistical average, $\mathbf{I}_{N}$ represents the $N \times N$ identity matrix; $\mathbf{d}[\mathbf{X}]$ represents the vector which is formulated by the diagonal entries of matrix $\mathbf{X}$. $\|\cdot\|$ denotes the Euclidean norm operator.

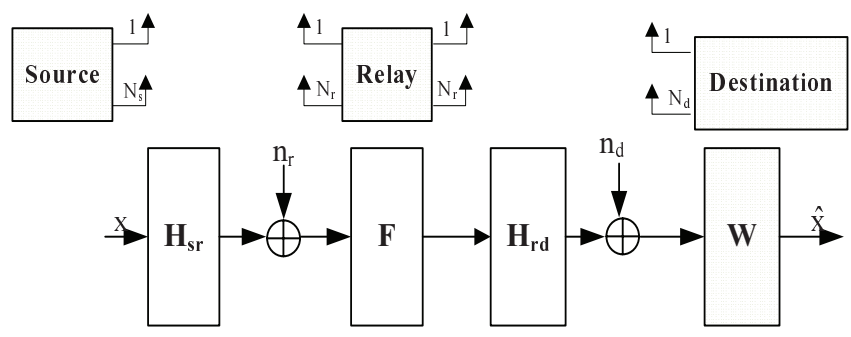

Fig. 1. MIMO Relay System Model.

\section{The Non-Regenerative MiMO Relay Model}

Let us consider a three-node relay communication model with source, relay and destination, as depicted in Fig.1. $N_{s}$, $N_{r}$ and $N_{d}$ are the number of antennas at the source, relay and the destination, respectively. Without loss of generality, it is assumed that there is no direct link between the source and the destination. MIMO relay works in half-duplex mode to avoid the mutual interference between its reception and transmission. The non-regenerative MIMO relay model works in the following two time slots mechanism:

The first time slot: Source node transmits signal to relay. And the received signal at relay is given by

$$
\mathbf{y}_{r}(t)=\mathbf{H}_{s r} \mathbf{x}(t)+\mathbf{n}_{r}(t) .
$$

where $E\left[\|\mathbf{x}(t)\|^{2}\right]=P_{s}, P_{s}$ represents the transmit power at source node. $\mathbf{n}_{r}(t)$ denotes the zero mean complex Gaussian noise with variances of $\sigma_{r}^{2}$ at the relay.

The second time slot: By using a predefined precoding matrix $\mathbf{F}$, relay regenerates the signal for destination based on the received signal from the source node. The received signal at destination will be

$$
\mathbf{y}_{d}(t+1)=\mathbf{H}_{r d} \mathbf{F} \mathbf{H}_{s r} \mathbf{x}(t)+\mathbf{H}_{r d} \mathbf{F} \mathbf{n}_{r}(t)+\mathbf{n}_{d}(t+1),
$$

where $\mathbf{n}_{d}(t+1)$ denotes the zero mean complex Gaussian noise with variances of $\sigma_{d}^{2}$ at the destination. $\mathbf{H}_{s r}$ and $\mathbf{H}_{r d}$ represent the channel matrix between source and relay, as well as the channel matrix between relay and destination, respectively. The design of precoding matrix $\mathbf{F}$ needs to satisfy the relay transmission power constraint, namely $E\left[\left\|\mathbf{F} \mathbf{y}_{r}(t)\right\|^{2}\right] \leq P_{r}$, where $P_{r}$ is the transmit power at relay.

\section{MSINR BASED MIMO RELAY PRECODING DESIGN}

It is shown in [9] that the destination may recover the transmitted signal $x(t)$ from the received signal $y_{d}(t+1)$ at the destination by using the Weiner filter as below

$$
\hat{\mathbf{x}}(t)=\mathbf{W}^{H} \mathbf{y}_{d}(t+1),
$$

where $\mathbf{W}=\left[\overline{\mathbf{H}} \overline{\mathbf{H}}^{H}+\overline{\mathbf{C}}\right]^{-1} \overline{\mathbf{H}}$, which is designed to achieve the minimal mean square error given by

$$
\begin{aligned}
& \mathbf{E}_{M S E}=E\left[(\hat{\mathbf{x}}(t)-\mathbf{x}(t))(\hat{\mathbf{x}}(t)-\mathbf{x}(t))^{H}\right] \\
& =\left[\mathbf{I}_{N_{s}}+\overline{\mathbf{H}}^{H} \overline{\mathbf{C}}^{-1} \overline{\mathbf{H}}\right]^{-1},
\end{aligned}
$$

where $\overline{\mathbf{H}}$ and $\overline{\mathbf{C}}$ represent the equivalent source-relaydestination channel and the equivalent noise covariance matrix, i.e.,

$$
\begin{gathered}
\overline{\mathbf{H}}=\mathbf{H}_{r d} \mathbf{F} \mathbf{H}_{s r}, \\
\overline{\mathbf{C}}=\sigma_{r}^{2} \mathbf{H}_{r d} \mathbf{F}\left(\mathbf{H}_{r d} \mathbf{F}\right)^{H}+\sigma_{d}^{2} \mathbf{I}_{N_{d}} .
\end{gathered}
$$

Now we can define the geometric product of the signal to interference plus noise ratio (GEOM-SINR) as [9]

$$
S I N R_{G E O M}=f\left(\mathbf{d}\left[\mathbf{E}_{M S E}\right]\right)=\prod_{i=1}^{N_{s}}\left(\frac{1}{\left[\mathbf{E}_{M S E}\right]_{i, i}}-1\right) .
$$

In this paper, we propose to use the above GEOM-SINR as the optimized criterion in the MIMO relay precoding design. Since the GEOM-SINR is dependent only on the diagonal entries of the MSE matrix of $\mathbf{E}_{M S E}$, the MSINR-based MIMO relay precoding design problem can be reformulated as the following optimization problem

$$
\begin{aligned}
& \arg \max _{\mathbf{F}} f\left(\mathbf{d}\left[\mathbf{E}_{M S E}\right]\right) \\
& \text { s.t. } \operatorname{tr}\left(\mathbf{F}\left[\mathbf{H}_{s r} \mathbf{H}_{s r}^{H}+\sigma_{r}^{2} \mathbf{I}_{N_{r}}\right] \mathbf{F}^{H}\right) \leq P_{r} .
\end{aligned}
$$

In order to derive the optimal precoding matrix $\mathbf{F}$ from the previous optimization problem, let us consider the SVD decomposition of the channel matrix of $\mathbf{H}_{s r}$ and $\mathbf{H}_{r d}$ as follows

$$
\begin{aligned}
& \mathbf{H}_{s r}=\mathbf{U}_{s r} \boldsymbol{\Lambda}_{s r} \mathbf{V}_{s r}, \\
& \mathbf{H}_{r d}=\mathbf{U}_{r d} \boldsymbol{\Lambda}_{r d} \mathbf{V}_{r d} .
\end{aligned}
$$

Without loss of generality, it is assumed that $N_{s} \leq$ $\min \left(\operatorname{rank}\left(\mathbf{H}_{s r}\right), \operatorname{rank}\left(\mathbf{H}_{r d}\right)\right)$, namely, the transmission requirement by the source can always be guaranteed by the MIMO relay system. It is known that the negative MSINR based objective function of $-f\left(\mathbf{d}\left[\mathbf{E}_{M S E}\right]\right)$ is a Schur concave function [8]. So it can be proved by following the majorization theory in [7] that the optimal MSINR based MIMO relay 
precoding matrix is given by (the detailed derivation can be found in Appendix)

$$
\mathbf{F}=\mathbf{V}_{r d, 1} \boldsymbol{\Lambda}_{f} \mathbf{U}_{s r, 1}^{H},
$$

where $\boldsymbol{\Lambda}_{f}$ is a $N_{s} \times N_{s}$ diagonal matrix, $\mathbf{V}_{r d, 1}$ and $\mathbf{U}_{s r, 1}$ denote the submatrix of $\mathbf{V}_{r d}$ and $\mathbf{U}_{s r}$, which corresponds to the $N_{s}$ strongest subchannels. It could be observed that, the MIMO relay precoding matrix $\mathbf{F}$ could be determined when we determine its diagonal entries.

Substituting (9) and (10) into (2) yields

$\tilde{\mathbf{y}}_{d}(t+1)=\boldsymbol{\Lambda}_{r d, 1} \boldsymbol{\Lambda}_{f} \boldsymbol{\Lambda}_{s r, 1} \tilde{\mathbf{x}}(t)+\boldsymbol{\Lambda}_{r d, 1} \boldsymbol{\Lambda}_{f} \tilde{\mathbf{n}}_{r}(t)+\tilde{\mathbf{n}}_{d}(t+1)$,

where $\tilde{\mathbf{x}}(t)=\mathbf{U}_{s r}^{H} \mathbf{x}(t), \tilde{\mathbf{y}}_{d}(t+1)=\mathbf{U}_{r d}^{H} \mathbf{y}_{d}(t+1), \tilde{\mathbf{n}}_{r}(t)=$ $\mathbf{U}_{s r}^{H} \mathbf{n}_{r}(t)$ and $\tilde{\mathbf{n}}_{d}(t+1)=\mathbf{U}_{r d}^{H} \mathbf{n}_{d}(t+1) . \boldsymbol{\Lambda}_{r d, 1}$ and $\boldsymbol{\Lambda}_{s r, 1}$ denote the diagonal submatrix of $\boldsymbol{\Lambda}_{r d}$ and $\boldsymbol{\Lambda}_{s r}$ with the $N_{s}$ largest entries. Obviously, the equivalent source-relaydestination link has been decomposed into $N_{s}$ parallel subchannels by utilizing MIMO relay precoding.

Let $\lambda_{s r, i}, \lambda_{r d, i}$ and $\lambda_{f, i}$ denote the $i$ th diagonal entry of the matrix of $\boldsymbol{\Lambda}_{s r}, \boldsymbol{\Lambda}_{r d}$ and $\boldsymbol{\Lambda}_{f}$, respectively. By substituting (9), (10) and (11) into (4) we obtain the diagonalized MSE matrix as follows

$$
\begin{aligned}
& \tilde{\mathbf{E}}_{M S E}=\left[\mathbf{I}_{N_{s}}+\right. \\
& \left.\left(\boldsymbol{\Lambda}_{r d, 1} \boldsymbol{\Lambda}_{f} \boldsymbol{\Lambda}_{s r, 1}\right)^{2}\left[\sigma_{r}^{2}\left(\boldsymbol{\Lambda}_{r d, 1} \boldsymbol{\Lambda}_{f}\right)^{2}+\sigma_{d}^{2} \mathbf{I}_{N_{s}}\right]^{-1}\right]^{-1} .
\end{aligned}
$$

Now the MSINR based MIMO relay precoding design problem can be reformulated as

$$
\begin{aligned}
& \arg \min _{\left\{x_{i}\right\}}-\sum_{i=1}^{N_{s}} \log _{10}\left(\frac{a_{i} b_{i} x_{i}+b_{i} x_{i}+a_{i}+1}{b_{i} x_{i}+a_{i}+1}-1\right) \\
& \text { s.t. } \sum_{i=1}^{N_{s}} x_{i} \leq P_{r}, x_{i} \geq 0,
\end{aligned}
$$

where

$$
a_{i}=\frac{\lambda_{s r, i}^{2}}{\sigma_{r}^{2}}, b_{i}=\frac{\lambda_{r d, i}^{2}}{\sigma_{d}^{2}}, x_{i}=\lambda_{f, i}^{2}\left(\lambda_{s r, i}^{2}+\sigma_{r}^{2}\right) .
$$

Now the MSINR-based MIMO relay precoding design problem is transformed into the power allocation problem. Since the optimization objective in (13) is now a concave function [7], the conventional convex optimization method could be utilized to derive the optimal solutions of $\lambda_{f, i}, i=1, \cdots, N_{s}$. According to the Karush-Kuhn-Tucker condition, the power waterfilling method can be applied to derive the closed-form solutions for $x_{i}, i=1, \cdots, N_{s}$ as follows

$$
x_{i}=\frac{\left[\sqrt{\left(a_{i}+1\right)^{2}+\frac{4\left(a_{i}+1\right) b_{i}}{v}}-\left(a_{i}+1\right)\right]^{+}}{2 b_{i}},
$$

where $[x]^{+}=\max (0, x)$, and $v$ could be determined by using the bisection method from the following formula

$$
\sum_{i=1}^{N_{s}} \frac{\left[\sqrt{\left(a_{i}+1\right)^{2}+\frac{4\left(a_{i}+1\right) b_{i}}{v}}-\left(a_{i}+1\right)\right]^{+}}{2 b_{i}}-P_{r}=0 .
$$

Now the diagonal entry $\lambda_{f, i}$ of $\Lambda_{f}$ can be calculated as

$$
\lambda_{f, i}=\sqrt{\frac{x_{i}}{\lambda_{s r, i}^{2}+\sigma_{r}^{2}}} .
$$

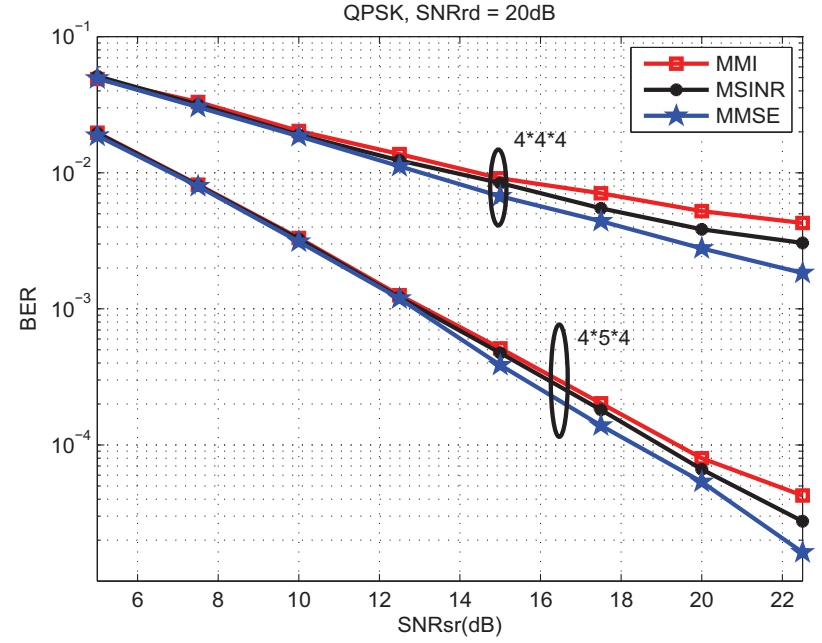

Fig. 2. The BER of different MIMO Relay Precoding with fixed $S N R_{r d}=$ $20 d B$.

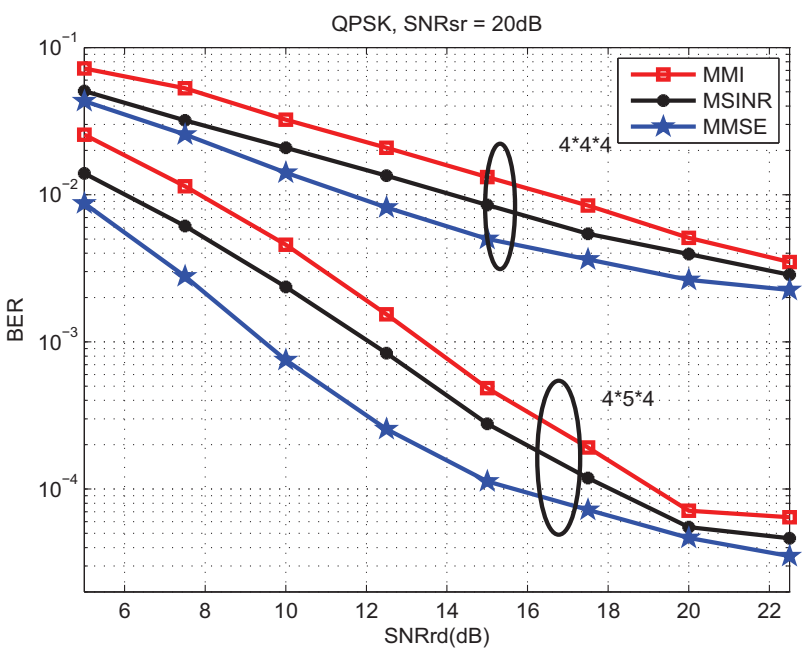

Fig. 3. The BER of different MIMO Relay Precoding with fixed $S N R_{s r}=$ $20 d B$.

\section{NUMERICAL RESULTS}

In order to validate the MSINR based MIMO relay precoding design, the bit error rate, mean square error, and the ergodic capacity of the MIMO relaying precoding are analyzed. The MMI-based MIMO relay precoding [3]- [4] and the MMSE-based MIMO relay precoding [5]- [6] will be used for comparison. Perfect channel estimate is assumed at both the relay and destination. Let $S N R_{s r}$ and $S N R_{r d}$ denote respectively the signal-to-noise ratio of the source-relay link and the relay-destination link, which are given by

$$
\begin{aligned}
& S N R_{s r}=\frac{P_{s}}{\sigma_{r}^{2} N_{s}}, \\
& S N R_{r d}=\frac{P_{r}}{\sigma_{d}^{2} N_{r}} .
\end{aligned}
$$




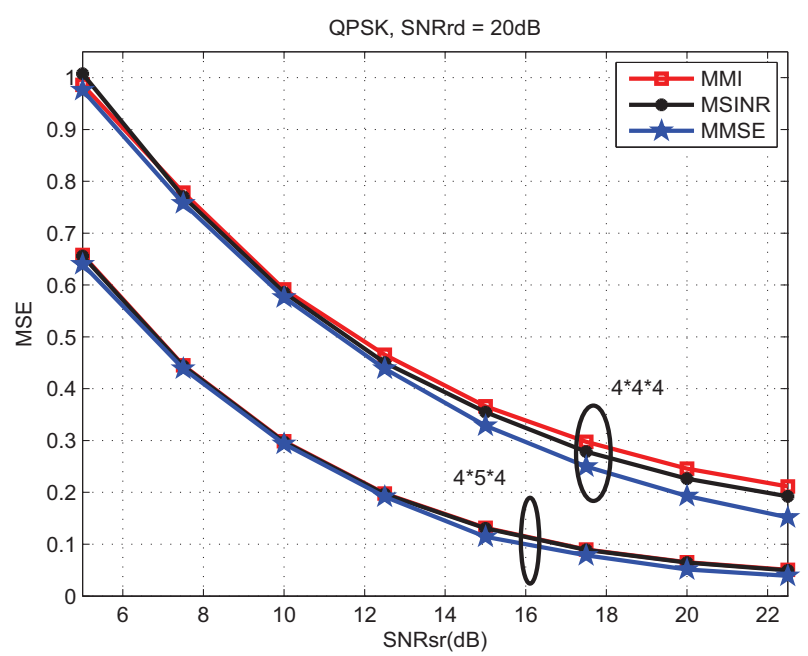

Fig. 4. The MSE of different MIMO Relay Precoding with fixed $S N R_{r d}=$ $20 d B$.

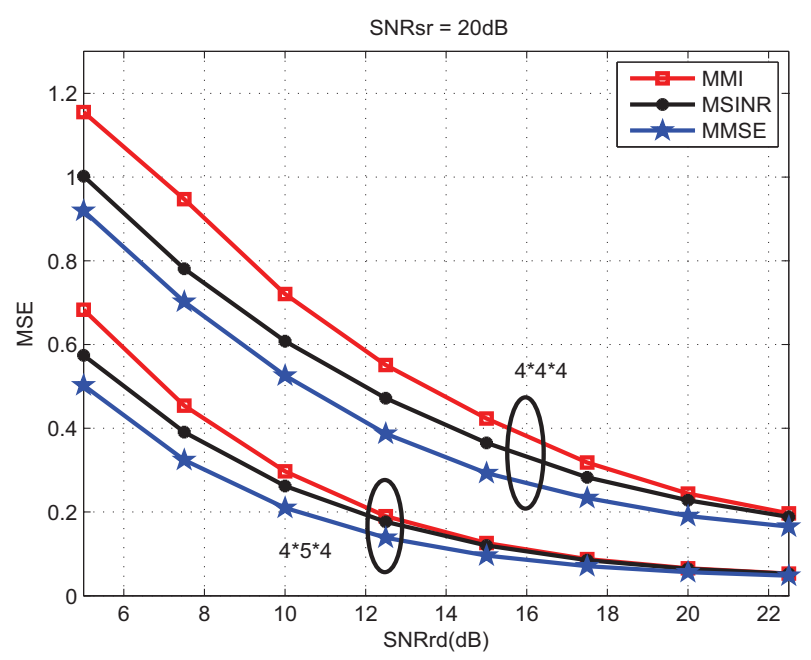

Fig. 5. The MSE of different MIMO Relay Precoding with fixed $S N R_{s r}=$ $20 d B$.

In our simulations, we employ QPSK modulation and assume that both source and destination have the same number of antennas, i.e, $N_{s}=N_{d}=4$. In order to examine the impact of the number of antennas at relay, we consider $N_{r}=4$ and $N_{r}=$ 5 in numerical simultaions. In all simulations, we assume $P_{s}=$ $N_{s}$ and $P_{r}=1$.

Firstly, the bit error rate (BER) performance of the MIMO relay system with different relay precoding schemes are illustrated in Fig. 2 and Fig. 3, where the fixed relaydestination link with $S N R_{r d}=20 d B$ and the fixed sourcerelay link with $S N R_{s r}=20 d B$ are assumed respectively. It can be observed that, the MMSE based precoding scheme outperforms the MMI based precoding scheme in terms of the MIMO relay precoding reliability. And the influence of both the source-relay link quality and the relay-destination

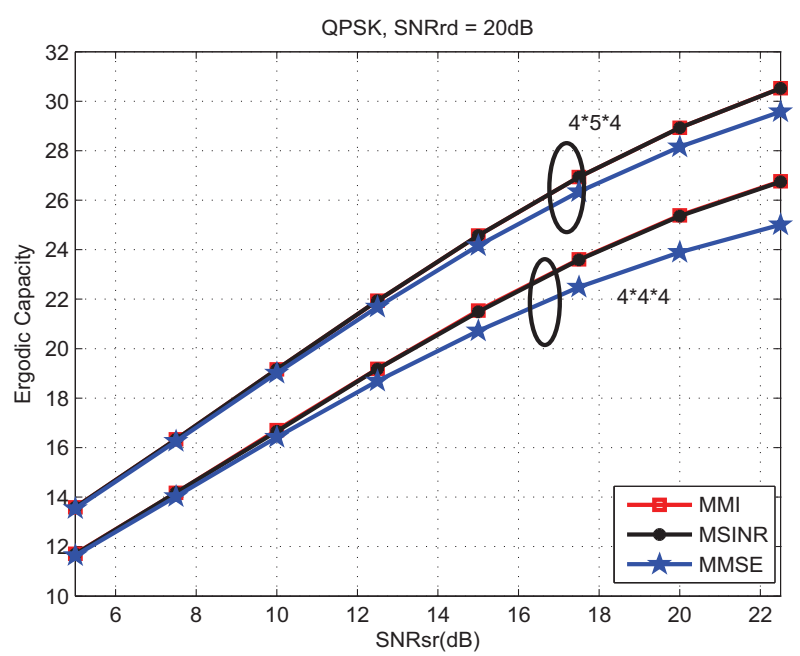

Fig. 6. The ergodic capacity of different MIMO Relay Precoding with fixed $S N R_{r d}=20 d B$.

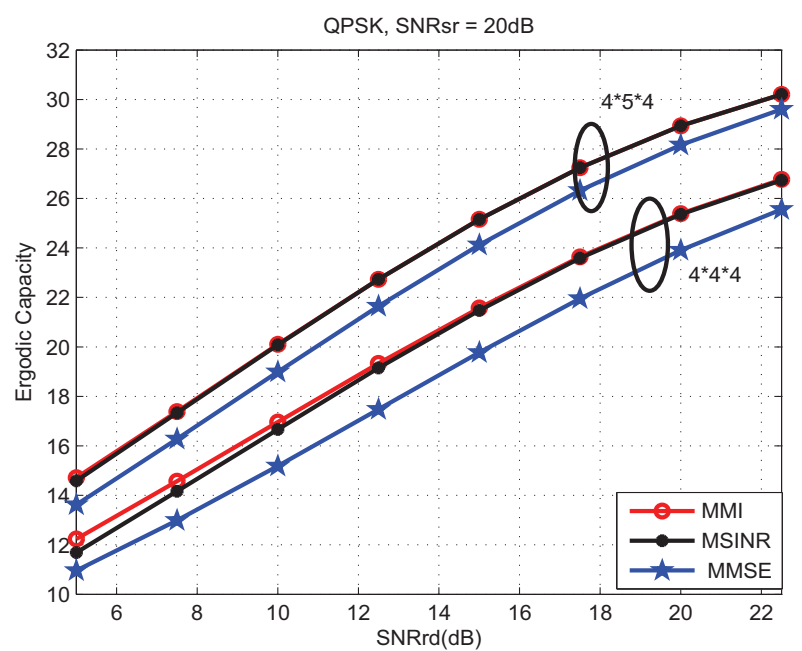

Fig. 7. The ergodic capacity of different MIMO Relay Precoding with fixed $S N R_{s r}=20 d B$.

link quality on the achieved reliability is presented in Fig. 2 and Fig. 3 as well. It could be readily observed that the inferior link in the MIMO relay system dominates the achieved performance. In addition, an increase in the number of antenna deployed at relay will lead to a significant improvement in the BER performance, which suggests that the reliability of the MIMO relay precoding scheme in an adverse environment can be compensated to a great extent by deploying more antennas at relay. It is noted that, the performance of the MSINR based MIMO relay precoding scheme lies between the MMSE-based precoding and the MMI-based precoding scheme. The simulated mean square error (MSE) of the MIMO relay system with different precoding schemes are illustrated in Fig. 4 and Fig. 5. Since the resulting BER directly depends on the equivalent SNR of the source-relay-destination link 


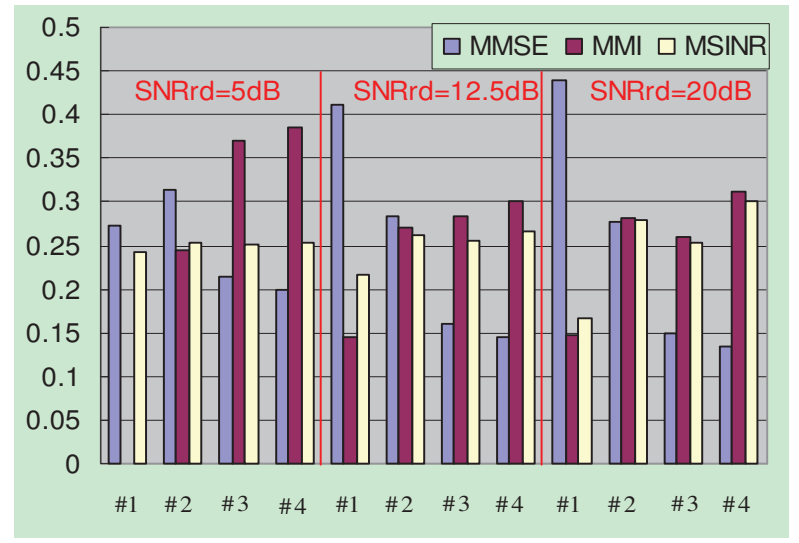

Fig. 8. The power allocation $\boldsymbol{\Lambda}_{f}$ among different subchannels with fixed $S N R_{s r}=20 d B$.

expressed in (7), one can observe similar characteristics in the MSE performance for three precoding schemes. The ergodic capacities of the MIMO relay system with different precoding schemes are illustrated in Fig. 6 and Fig. 7. As expected, the MMI based precoding scheme outperforms the MMSE based precoding in terms of the ergodic capacity. It is noted that, within moderate and high SNR region, the MSINR based precoding can realize almost the same ergodic capacity as that achieved by using the MMI based precoding scheme.

In order to further explain the reason why three MIMO relay precoding schemes achieve different reliabilities and ergodic capacities, the power allocation among sub-channels after the relay precoding in the three MIMO relay precoding schemes are illustrated in Fig. 8, where the fixed source-relay link with $S N R_{s r}=20 \mathrm{~dB}$ is assumed. And the four eigenvalues of $\tilde{\boldsymbol{\Lambda}}=\boldsymbol{\Lambda}_{s r, 1} \boldsymbol{\Lambda}_{r d, 1}$ are $\tilde{\lambda}_{1}=0.046, \tilde{\lambda}_{2}=2.92, \tilde{\lambda}_{3}=22.44, \tilde{\lambda}_{4}=$ 88.62 , which are marked respectively with $\# 1, \# 2, \# 3$ and \#4 in Fig. 8. It can be observed that, due to their different optimized criteria, the MMI-based precoding and the MMSEbased precoding will have different power allocation policy among different subchannels. The MMSE-based precoding tends to allocate more power to the less reliable sub-channel to guarantee the transmission reliability. On the other hand, the MMI-based precoding prefers to allocate more power to the more reliable sub-channel to improve the ergodic capacity. In fact, these two different power allocation strategies make two precoding schemes achieve different levels of reliability and ergodic capacity. It is also shown that, with the MSINRbased precoding scheme, the power allocation among different subchannels is approximately uniform within low $S N R_{r d}$ region. While the power allocation tends to converge to that utilized by the MMI-based MIMO relay precoding within moderate and high $S N R_{r d}$ regions. This explicates why the MSINR based MIMO relay precoding achieves the reliability and the ergodic capacity in between the MMI-based MIMO relay precoding scheme and the MMSE-based MIMO relay precoding scheme.

\section{CONCLUding REMARKS}

MIMO relay technique effectively combines the advantages of both the MIMO and relay technologies. Motivated by the recent progress in the non-regenerative MIMO relay precoding, we have proposed to maximize the GEOM-SINR to derive the MSINR based MIMO relay precoding scheme. Our analysis unveils that, compared with the MMI-based precoding design and the MMSE-based precoding design, the MSINR based MIMO precoding offers a better tradeoff between the communication reliability and the realized system ergodic capacity, which makes it an attractive alternative in nonregenerative MIMO relay communication systems.

\section{APPENDIX}

According to [7], we have the following Lemmas.

Lemma 1 Given a hermitian matrix $\mathbf{A}, \mathbf{d}[\mathbf{A}]$ represents the vector of main diagonal elements $\bar{\lambda}[\mathbf{A}], \mathbf{d}[\mathbf{A}] \prec \bar{\lambda}[\mathbf{A}] . \mathbf{x} \prec \mathbf{y}$ means that vector $\mathbf{x}$ is majorized by vector $\mathbf{y}$.

Lemma 2 For two complex $N \times N$ matrix $\mathbf{A}_{1}$ and $\mathbf{A}_{2}$, let $\mathbf{B}=\mathbf{A}_{1}^{H} \mathbf{A}_{2} \mathbf{A}_{1}$, then $\bar{\sigma}_{b} \prec w\left(\bar{\sigma}_{a_{1}} \odot \bar{\sigma}_{a_{2}} \odot \bar{\sigma}_{a_{1}}\right)$, where $\bar{\sigma}_{b}, \bar{\sigma}_{a_{1}}$ and $\bar{\sigma}_{a_{2}}$ represent, respectively, the $N \times 1$ vectors containing the singular values of the matrix $\mathbf{B}, \mathbf{A}_{1}$ and $\mathbf{A}_{2}$ arranged in the same order. $\odot$ denotes the Schur (elementwise) product of two vectors. $\mathbf{x} \prec_{w} \mathbf{y}$ means that vector $\mathbf{x}$ is weakly majorized by vector $\mathbf{y}$.

Lemma $\mathbf{3} \mathbf{x} \prec_{w} \mathbf{y} \Rightarrow f(\mathbf{x}) \leq f(\mathbf{y})$ if and only if $f(\cdot)$ is an increasing and Schur convex function.

Lemma 4 For two $N \times N$ semi-definite positive hermitian matrices $\mathbf{A}$ and $\mathbf{B}, \lambda_{a_{i}}$ and $\lambda_{b_{i}}$ denotes the $i$ th eigenvalue of $\mathbf{A}$ and $\mathbf{B}$ with the same ordering, $\operatorname{tr}(\mathbf{A B}) \geq \sum_{i=1}^{N} \lambda_{a, i} \lambda_{b, N+1-i}$. To prove the optimal MIMO relay precoding matrix $\mathbf{F}$ in (10), let us denote

$$
\begin{aligned}
& \mathbf{A}=\mathbf{H}_{s r} \mathbf{H}_{s r}^{H}=\mathbf{U}_{a} \boldsymbol{\Lambda}_{a} \mathbf{U}_{a}^{H}, \\
& \mathbf{X}=\mathbf{H}_{r d} \mathbf{F}\left(\mathbf{A}+\sigma_{r}^{2} \mathbf{I}_{N_{r}}\right)^{1 / 2}=\mathbf{U}_{x} \boldsymbol{\Lambda}_{x} \mathbf{V}_{x}^{H},
\end{aligned}
$$

where $\boldsymbol{\Lambda}_{a}$ represents the nonzero diagonal eigenvalue matrix of $\mathbf{A}$. Here it is assumed that the diagonal eigenvalues in $\boldsymbol{\Lambda}_{a}$ and $\boldsymbol{\Lambda}_{x}$ are in ascending order. According to the matrix theory, we have

$$
\begin{aligned}
& \mathbf{H}_{s r}=\mathbf{U}_{a} \boldsymbol{\Lambda}_{a}^{1 / 2} \mathbf{Q}_{1}, \\
& \mathbf{H}_{r d} \mathbf{F}=\mathbf{X}\left(\mathbf{A}+\sigma_{r}^{2} \mathbf{I}_{N_{r}}\right)^{-1 / 2},
\end{aligned}
$$

where $\mathbf{Q}_{1}$ represents any unitary matrix. By substituting (22) and (23) into (4), we can rewrite the MSE matrix in (24), where we have used the matrix inverse relationship of $(\mathbf{I}+$ $\left.\mathbf{A}^{-1}\right)^{-1}=\mathbf{I}-(\mathbf{A}+\mathbf{I})^{-1}$ to derive the first equality, and

$$
\begin{aligned}
& \mathbf{Q}_{2}=\mathbf{V}_{x}^{H} \mathbf{U}_{a}, \mathbf{D}_{1}=\left(\boldsymbol{\Lambda}_{a}^{-1}+\sigma_{r}^{2} \mathbf{I}_{N_{s}}\right)^{-1 / 2}, \\
& \mathbf{D}_{2}=\left(\boldsymbol{\Lambda}_{x}^{-2}+\sigma_{d}^{2} \mathbf{I}_{N_{s}}\right)^{-1} .
\end{aligned}
$$

Based on the Lemma 1 and Lemma 2, we have

$$
\mathbf{d}[\mathbf{G}] \prec \bar{\lambda}[\mathbf{G}] \prec_{w} \mathbf{d}\left[\mathbf{D}_{1}^{2} \mathbf{D}_{2}\right],
$$

which implies that, $\mathbf{d}[\mathbf{G}]$ will be maximized when $\mathbf{Q}_{1}=$ $\boldsymbol{\Phi}_{N_{s}}, \mathbf{Q}_{2}=\boldsymbol{\Phi}_{N_{s}}$, where $\boldsymbol{\Phi}_{N_{s}}$ represents the diagonal matrix with unity-norm entry, namely, $\left\|\left[\mathbf{\Phi}_{m}\right]_{i, i}\right\|=1,\left\|\left[\boldsymbol{\Phi}_{m}\right]_{i, j}\right\|=0$ for $i, j=1, \cdots, m, i \neq j$.

Now that the negative objective function of $-f\left(\mathbf{d}\left[\mathbf{E}_{M S E}\right]\right)$ in (7) is a Schur concave function, which is also an increasing function of variable $\mathbf{d}\left[\mathbf{E}_{M S E}\right],-f\left(\mathbf{d}\left[\mathbf{I}_{N_{s}}-\mathbf{G}\right]\right)$ is also a Schur 


$$
\begin{aligned}
& \left.\tilde{\mathbf{E}}_{M S E}=\mathbf{I}_{N_{s}}-\overline{\mathbf{H}}^{H}\left[\mathbf{H}_{r d} \mathbf{F}\left(\mathbf{A}+\sigma_{r}^{2} \mathbf{I}_{N_{r}}\right)\left(\mathbf{H}_{r d} \mathbf{F}\right)^{H}\right)+\sigma_{d}^{2} \mathbf{I}_{N_{d}}\right]^{-1} \overline{\mathbf{H}} \\
& =\mathbf{I}_{N_{s}}-\mathbf{Q}_{1}^{H} \Lambda_{a}^{1 / 2} \mathbf{U}_{a}^{H}\left(\mathbf{U}_{a} \boldsymbol{\Lambda}_{a} \mathbf{U}_{a}^{H}+\sigma_{r}^{2} \mathbf{I}_{N_{s}}\right)^{-1 / 2} \mathbf{V}_{x} \boldsymbol{\Lambda}_{x} \mathbf{U}_{x}^{H}\left[\mathbf{U}_{x} \boldsymbol{\Lambda}_{x}^{2} \mathbf{U}_{x}^{H}+\sigma_{d}^{2} \mathbf{I}_{N_{s}}\right]^{-1} \boldsymbol{\Lambda}_{x} \mathbf{V}_{x}^{H}\left(\mathbf{U}_{a} \boldsymbol{\Lambda}_{a} \mathbf{U}_{a}^{H}+\sigma_{r}^{2} \mathbf{I}_{N_{s}}\right)^{-1 / 2} \mathbf{U}_{a} \boldsymbol{\Lambda}_{a} \mathbf{Q}_{1} \\
& =\mathbf{I}_{N_{s}}-\mathbf{Q}_{1}^{H} \mathbf{D}_{1} \mathbf{Q}_{2}^{H} \mathbf{D}_{2} \mathbf{Q}_{2} \mathbf{D}_{1} \mathbf{Q}_{1}=\mathbf{I}_{N_{s}}-\mathbf{G} .
\end{aligned}
$$

$$
\begin{gathered}
\mathbf{X}=\mathbf{H}_{r d} \mathbf{F}\left(\mathbf{A}+\sigma_{r}^{2} \mathbf{I}_{N_{r}}\right)^{1 / 2}=\mathbf{U}_{x} \boldsymbol{\Lambda}_{x} \mathbf{V}_{x}^{H} \Rightarrow \mathbf{U}_{r d, 1} \boldsymbol{\Lambda}_{r d, 1} \mathbf{V}_{r d, 1}^{H} \mathbf{F}=\mathbf{U}_{r d, 1} \boldsymbol{\Lambda}_{x} \mathbf{U}_{s r, 1}^{H}\left(\mathbf{U}_{s r, 1} \boldsymbol{\Lambda}_{a} \mathbf{U}_{s r, 1}^{H}+\sigma_{r}^{2} \mathbf{I}_{N_{r}}\right)^{-1 / 2} \Rightarrow \\
\mathbf{U}_{r d, 1} \boldsymbol{\Lambda}_{r d, 1} \mathbf{V}_{r d, 1}^{H} \mathbf{F}=\mathbf{U}_{r d, 1} \boldsymbol{\Lambda}_{x}\left(\mathbf{U}_{s r, 1}^{-1} \boldsymbol{\Lambda}_{a}\left(\mathbf{U}_{s r, 1}^{H}\right)^{-1}+\sigma_{r}^{2}\left(\mathbf{U}_{s r, 1}^{H} \mathbf{U}_{s r, 1}\right)^{-2}\right)^{-1 / 2} \mathbf{U}_{s r, 1}^{H} \Rightarrow \\
\mathbf{U}_{r d, 1} \boldsymbol{\Lambda}_{r d, 1} \mathbf{V}_{r d, 1}^{H} \mathbf{F}=\mathbf{U}_{r d, 1} \boldsymbol{\Lambda}_{x}\left(\boldsymbol{\Lambda}_{a}+\sigma_{r}^{2} \mathbf{I}_{N_{s}}\right)^{-1 / 2} \mathbf{U}_{s r, 1}^{H}
\end{gathered}
$$

concave function, which is a decreasing function of variable $\mathbf{d}[\mathbf{G}]$. Obviously, $f\left(\mathbf{d}\left[\mathbf{I}_{N_{s}}-\mathbf{G}\right]\right)$ is an increasing Schur convex function with respect to the variable $\mathbf{d}[\mathbf{G}]$. So we have the following relationship based on (26) and Lemma 3

$$
-f\left(\mathbf{d}\left[\mathbf{I}_{N_{s}}-\mathbf{G}\right]\right) \geq-f\left(\mathbf{d}\left[\mathbf{D}_{1}^{2} \mathbf{D}_{2}\right]\right) .
$$

Without changing the original objective function and the transmit power constraint, we can choose $\mathbf{Q}_{1}=\mathbf{Q}_{2}=\mathbf{I}_{N_{s}}$ to transform the previous inequality into an equality such that $\mathbf{V}_{x}=\mathbf{U}_{a}$. Now let us consider the transmit power constraint at relay. Firstly, by substituting the SVD decomposition of $\mathbf{H}_{r d}$ into (24), we have

$$
\begin{array}{r}
{\left[\begin{array}{cc}
\mathbf{0}_{\left(N_{d}-r_{2}\right) \times\left(N_{r}-r_{2}\right)} & \mathbf{0}_{\left(N_{d}-r_{2}\right) \times r_{2}} \\
\mathbf{0}_{r_{2} \times\left(N_{r}-r_{2}\right)} & \boldsymbol{\Lambda}_{r d, r_{2}}
\end{array}\right] \hat{\mathbf{F}}} \\
=\mathbf{U}_{r d}^{H} \mathbf{X}\left(\mathbf{A}+\sigma_{r}^{2} \mathbf{I}_{N_{r}}\right)^{-1 / 2}
\end{array}
$$

where $r_{2}=\operatorname{rank}\left(\mathbf{H}_{r d}\right)$. The diagonal matrix of $\boldsymbol{\Lambda}_{r d, r_{2}}$ consists of all non-zero eigenvalues of matrix $\mathbf{H}_{r d}$, and $\hat{\mathbf{F}}=\mathbf{V}_{r d}^{H} \mathbf{F}$. Let $\mathbf{U}_{r d}=\left[\mathbf{U}_{r d, \bar{r}_{2}}, \mathbf{U}_{r d, r_{2}}\right]$, where $\mathbf{U}_{r d, \bar{r}_{2}}$ and $\mathbf{U}_{r d, r_{2}}$ comprises of the eigen-vectors in $\mathbf{U}_{r d}$ with zero singular values and non-zero singular values, respectively. Since $N_{s} \leq \min \left(\operatorname{rank}\left(\mathbf{H}_{s r}, \mathbf{H}_{r d}\right)\right)$, we may assign $\mathbf{U}_{r d, r_{2}}=\mathbf{U}_{r d, 1}$, $\boldsymbol{\Lambda}_{r d, r_{2}}=\boldsymbol{\Lambda}_{r d, 1}$. Now let us consider three cases to satisfy the equality in (28).

- If $N_{d}=N_{r}=r_{2}$, (28) is satisfied if and only if

$$
\hat{\mathbf{F}}=\boldsymbol{\Lambda}_{r d, r_{2}}^{-1} \mathbf{U}_{r d, r 2}^{H} \mathbf{U}_{x} \boldsymbol{\Lambda}_{x} \mathbf{V}_{x}^{H}\left(\mathbf{A}+\sigma_{r}^{2} \mathbf{I}_{N_{r}}\right)^{-1 / 2} .
$$

- If $N_{d}>N_{r}=r_{2}$, (28) holds if and only if $\mathbf{U}_{r d, \bar{r}_{2}}^{H} \mathbf{U}_{x}=$ $\mathbf{0}_{\left(N_{d}-r_{2}\right) \times N_{r}}$ and (29) is satisfied.

- If $N_{d}>r_{2}, N_{r}>r_{2}$, (28) holds if and only if $\mathbf{U}_{r d, \bar{r}_{2}}^{H} \mathbf{U}_{x}=\mathbf{0}_{\left(N_{d}-r_{2}\right) \times N_{s}}$ and

$$
\begin{aligned}
\hat{\mathbf{F}}=\left(\mathbf{0}_{r_{2} \times\left(N_{r}-r_{2}\right)}\right. & \left.\boldsymbol{\Lambda}_{r d, r_{2}}^{-1}\right)^{H} \mathbf{U}_{r d, r 2}^{H} \mathbf{U}_{x} \boldsymbol{\Lambda}_{x} \\
& \mathbf{V}_{x}^{H}\left(\mathbf{A}+\sigma_{r}^{2} \mathbf{I}_{N_{r}}\right)^{-1 / 2} .
\end{aligned}
$$

Considering the previous three cases, the transmit power at relay can be expressed as

$$
\begin{aligned}
& \operatorname{tr}\left(\mathbf{F}\left(\mathbf{A}+\sigma_{r}^{2} \mathbf{I}_{N_{r}}\right) \mathbf{F}^{H}\right)=\operatorname{tr}\left(\hat{\mathbf{F}}\left(\mathbf{A}+\sigma_{r}^{2} \mathbf{I}_{N_{r}}\right) \hat{\mathbf{F}}^{H}\right) \\
& \quad=\operatorname{tr}\left(\boldsymbol{\Lambda}_{r d, r_{2}}^{-1} \mathbf{U}_{r d, r_{2}}^{H} \mathbf{U}_{x} \boldsymbol{\Lambda}_{x}^{2} \mathbf{U}_{x}^{H} \mathbf{U}_{r d, r_{2}} \boldsymbol{\Lambda}_{r d, r_{2}}^{-1}\right)
\end{aligned}
$$

Based on Lemma 4 , if $\mathbf{U}_{x}=\mathbf{U}_{r d, r_{2}} \boldsymbol{\Phi}_{N_{s}}=\mathbf{U}_{r d, 1} \boldsymbol{\Phi}_{N_{s}}$, (31) will have the minimum. In order not to violate the original objective function and the transmit power constraint, let $\mathbf{U}_{x}=$ $\mathbf{U}_{r d, 1}$ and $\mathbf{V}_{x}=\mathbf{U}_{a}=\mathbf{U}_{s r, 1}$, (21) can be reformulated to (32).
Now we can conclude that the optimal MSINR based MIMO relay precoding matrix $\mathbf{F}$ is given by

$$
\mathbf{F}=\mathbf{V}_{r d, 1} \boldsymbol{\Lambda}_{r d, 1}^{-1} \boldsymbol{\Lambda}_{x}\left(\boldsymbol{\Lambda}_{a}+\sigma_{r}^{2} \mathbf{I}_{N_{s}}\right)^{-1 / 2} \mathbf{U}_{s r, 1}^{H}=\mathbf{V}_{r d, 1} \boldsymbol{\Lambda}_{f} \mathbf{U}_{s r, 1}^{H} .
$$

\section{REFERENCES}

[1] G.J.Foschini, M. Gans, "On limits of wireless communications in a fading environment when using multiple antennas," Wireless Personal Commun., vol. 6, pp. 311-335, 1998.

[2] S. Simoens, O. Munoz, J. Vidal, and A. del Coso, "On the Gaussian MIMO relay channel with full channel state information," IEEE Trans. Signal Process., vol. 57, pp. 3588-3599, Sep. 2009.

[3] X.Tang, Y. Hua, "Optimal design of non-regenerative MIMO wireless relays," IEEE Trans. Wireless Commun., vol. 6, pp. 1398-1407, 2007.

[4] O.Munoz-Medina, J. Vidal, A. Agustin, "Linear transceiver design in nonregenerative relays with channel state information," IEEE Trans. Signal Process, vol. 55, no. 6, pp. 2593-2604, 2007.

[5] W.Guan, H.Luo, "Joint MMSE transceiver design in non-regenerative MIMO relay systems," IEEE Commun. Lett., vol. 12, pp. 517-519, 2008.

[6] A. S. Behbahani, R. Merched, A. M. Eltawil, "Optimizations of a MIMO relay network," IEEE Trans. Signal Process., vol. 56, no. 10, pp. 50625073, 2008.

[7] A.W. Marshall, I. Olkin, Inequalities: Theory of Majorization and Its Applications, New York: Academic, 1979.

[8] D. P. Palomar, J. M. Cioffi, M. A. Lagunas, "Joint Tx-Rx beamforming design for multicarrier MIMO channels: A unified framework for convex optimization," IEEE Trans. Signal Process., vol. 51, no. 9, pp. 2381-2401, 2003.

[9] Y. Rong, X. Tang, Y. Hua, "A unified framework for optimizing linear non-regenerative multicarrier MIMO relay communication systems," IEEE Trans. Signal Process., vol. 57, no.12, pp. 4837-4851,2009. 